\title{
Popularity Proportional Cache Size Allocation Policy for Video Delivery on CCN
}

\author{
Haipeng Li, Hidenori Nakazato \\ Global Information and Telecommunication Studies, \\ Waseda University \\ Tokyo, Japan \\ \{freebirdlee@ruri.,nakazato@\}waseda.jp
}

\author{
Andrea Detti, Nicola Blefari Melazzi \\ CNIT, Department of Electronic Engineering, \\ University of Rome "Tor Vergata" \\ Rome, Italy \\ \{andrea.detti, blefari\}@uniroma2.it
}

\begin{abstract}
Content Centric Networking (CCN), which is regarded as an innovational architecture of future network, is increasingly gaining attention in recent years. Based on the observation of current Internet usage, $\mathrm{CCN}$ presents a contentcentric model which focuses on data distribution and retrieval. To enhance the efficiency of content distribution, In-network caching is adopted, which enables routers to temporarily store the passing by data. Thus the scheme of cache management will affect the network performance significantly. In this paper, a cache replacement policy named Popularity Proportional Cache Size Allocation (PPCSA) policy has been proposed, which designed for video content delivery on CCN. Unlike other previous works in this area, which have focused on the popularity of individual content chunks, in this study, we not only consider the title-level popularity of a video which is composed of a set of video segments, but also take the chunk-level probability of video segments within a video title into consideration. We evaluated our policy through simulations, by using a simple 4-level tree topology and a real network based hybrid topology. The results validate that on both scenarios, the proposed approach can increase the average cache hit ratio and shorten the average content delivery distance between requesters and object contents. In addition, we analyzed the energy efficiency of PPCSA policy, and we found that compared to other three cache replacement policies, Least Recently Used (LRU), Least Frequently Used (LFU) and First in First out (FIFO), PPCSA reduced the transmission energy consumption, and improved the energy efficiency in idle time.
\end{abstract}

Keywords-CCN; video delivery; cache; popularity;PPCSA

\section{INTRODUCTION}

According to the latest white paper of Cisco Visual Networking Index (CVNI) [1], the global Internet traffic is growing extremely. The increasing data traffic of content sharing and acquiring, especially for high quality video streaming, is the primary contributor to this worldwide network traffic growth. And the usage of current network has gradually evolved from host to host communication which was appropriate for the needs of the early Internet, to large scale content sharing and delivery. Due to the limitation of the current IP network model, new types of network architectures have been proposed to address this challenge [2] - [4].

Content Centric Networking (CCN) [5], one of the proposals of future network architecture, has emerged in recent years, and rapidly gained wide attention of researchers. Inspired by the future needs of the Internet, $\mathrm{CCN}$ adopts content name based routing instead of IP address based routing, and focuses on distributing and acquiring contents rather than end-host communication. To promote efficient content delivery, in-network caching has been deployed in $\mathrm{CCN}$ which allows the contents being transferred to be cached in the intermediate routers. This makes the subsequent users be able to obtain these content copies from the closer routers without visiting the original source.

With the features of in-network caching, $\mathrm{CCN}$ is capable of facilitating multicast of contents, and shortening the content delivery distance between content copies and requesters, by which the unnecessary network traffic can be reduced. Thus an appropriate cache management scheme could improve the network performance greatly.

In this paper, we propose a cache replacement strategy named Popularity Proportional Cache Size Allocation policy (PPCSA), for improving the cache performance of video delivery on $\mathrm{CCN}$. Based on the features of video content request model, we not only considered the title-level popularity of each video file which consists of a group of video segments, but also took the chunk-level probability of video segments into account. PPCSA has been evaluated in our simulation on two topologies with variable cache sizes. And the results show that compared with LRU, LFU and FIFO, our proposal enhances the network performance of $\mathrm{CCN}$ in serval aspects. In addition, the result of energy efficiency analysis indicates that PPCSA is more energy efficient compared to the other three cache replacement policies.

The remainder of this paper is organized as follows: Section II discusses the related work of cache management schemes on $\mathrm{CCN}$ and cache replacement policies for video delivery. In Section III we describe our proposed cache replacement policy PPCSA. In Section IV we present our simulation and its results as well as energy efficiency analysis. Finally, a brief summary and our conclusions are provided in Section V.

\section{RELATED WORKS}

By suggesting the new architecture of future network, CCN provides various challenges for researchers to study. And there 
are many researches existing in the study of cache strategy and video delivery.

\section{A. Cache management schemes for CCN}

A holistic model [6] has been developed to analyze the performance and overhead of cache management strategies in CCN. Based on this model, they proposed an optimal cache strategy named Coordinating In-network Caching to improve the overall $\mathrm{CCN}$ network performance and optimize the cache management cost. An in-network caching scheme ProbCache [7] has been introduced, which aims to reduce the cache redundancy on the content transmission path, and fairly distributes contents for multiple requesters on this path. Martin Draxler and Holger Karl proposed off-path based cache strategies [8] to avoid redundant caching of contents, so as to improve the utilization of cache space.

These above mentioned researches contribute to improve the cache efficiency of $\mathrm{CCN}$ in various aspects. However the request object of their studies is the general contents. As for multiplexing a set of ordered contents e.g. video segments, the cache management scheme needs to be reconsidered.

\section{B. Cache replacement policy for video delivery}

Cache replacement policy aims to carefully choose an appropriate content in CS to evict, in order to make room for the new incoming contents. Although many efficiency cache replacement policies such as LRU, LFU, FIFO have been widely adopted, the researches of seeking more efficient policy of $\mathrm{CCN}$ is being carried out.

Two cache replacement algorithms have been propose to enhance the efficiency of H.264/SVC based video streaming delivered in $\mathrm{CCN}$ [9], while the scalability of these algorithms is under discussion. A Reuse time (RT) [10] based caching policy has been presented, which is a practical extension study of MIN algorithm [11]. RT caching policy exploits the periodicity of request stream pattern in the case of video streaming, and exactly predicts the reuse time of a segment by the knowledge of play start time of each user to view this video. However, the overhead of predicting reuse times for every video segment has not been clearly assessed.

\section{Popularity Proportional CACHE Size Allocation POLICY}

\section{A. Assumptions of video request model in CCN}

In $\mathrm{CCN}$, contents are requested according to its individual names, and the name structure used in $\mathrm{CCN}$ is hierarchical name structure, e.g. /Prefix/Videoi/Contentj. Generally, a video file in $\mathrm{CCN}$ is composed of a set of video segments (chunks) which are requested in sequence while video is being played.

We therefore assume that the name of requested video chunks in CCN includes a video title name $i$ and a sequence number $j$ which used for distinguishing different segments of this video. And the sequence number $j$ is ordered based on video playback time. Accordingly, we define that a video file $f_{i}$ consists of a set of video segments $\left\{C_{i, 1}, C_{i, 2}, \ldots, C_{i, j} \ldots\right\}$, which are ordered by its sequence number $j$. Besides, in this study we consider that videos will be requested from the first segment to the last one.

\section{B. The PPCSA model}

PPCSA is a cache replacement strategy which we concerned with both title-level popularity of videos and chunklevel popularity of video segments. Moreover, based on the natural linear time structure of video segments, the popularity of each segment within a video refers to the probability of this content being requested in the future. The PPCSA model can be divided into two components: Cache Size Allocation and Evictee Selection, and the two types of popularity mentioned above are adopted respectively.

\section{1) Cache Size Allocation}

We argue that according to the title-level popularity, the allocation of storage space for each video in the CS needs to rapidly response to the dynamic variation of request ratio. Thus, we measure the request ratio $R e q_{i}$ for each title and calculate the real time CS occupancy rate $R c s_{i}$ for video title $i$ cached in the CS, in order to accurately adjust the allocated cache size for each video while cache replacement occurred. And the calculation of $\operatorname{Req}_{i}$ and $R c s_{i}$ takes place as follows:

$$
\begin{aligned}
R e q_{i} & =\frac{\sum_{j=1}^{n} \operatorname{Rec}_{i j}}{\sum_{i=1}^{m} \sum_{j=1}^{n} \operatorname{Rec}_{i j}} \\
R c s_{i} & =\frac{\sum_{j=1} R c s_{i j}}{K}
\end{aligned}
$$

$\operatorname{Rec}_{i j} \in\{0,1,2, \ldots\}$ represents the observed number of requests for content $C_{i, j}$ in a single time unit. The $m, n$ in (1), are considered as the amount of requested video titles and the number of requested segments respectively. According to (1), request rate $R e q_{i}$ implies the dynamic popularity of video title $f_{i}$

In (2), $R c s_{i j} \in\{0,1\}$, indicates whether content $C_{i, j}$ is cached in this CS, and $K$ is the CS cache capacity of a node. Therefore, the real time cache occupancy ratio for video $f_{i}$ in CS can be expressed by (2). Namely, $\operatorname{Rcs}_{i}$ represents the proportion of cache size occupied by video file $f_{i}$.

Our goal is to allocate cache proportional to content popularity, i.e., $R e q_{i}=R c s_{i}$. $R e q_{i}<R c s_{i}$ indicates that file $f_{i}$ is occupying more cache spaces than it needs. On the contrary, $R e q_{i}>R c s_{i}$, we can regard that more contents of video $f_{i}$ need to be cached to meet the request ratio, in order to enhance the utilization of CS.

\section{2) Evictee selection}

Video viewers request video segments in the order of content sequence number $j$, then we deduce that the subsequent segments have higher probability to be requested in the future. For instance, if a CCN router receives a request for content $C_{i, 6}$, the subsequent contents of the same video such as $C_{i, 7}, C_{i, 8}$ etc. would highly be requested later. Therefore, any of these subsequent contents which cached in CS, will have a higher opportunity to be requested than prior contents. And PPCSA aims to keep these subsequent contents in the CS for the future requests by selecting the content with the smallest sequence number $j$ to be the evictee candidate. 


\section{PPCSA cache replacement policy}

PPCSA cache replacement policy is able to adjust the CS space occupied by each video file due to the consideration of title-level popularity while processing cache replacement algorithm. In the meantime, PPCSA policy evicts the content which has smallest probability to be requested in the future.

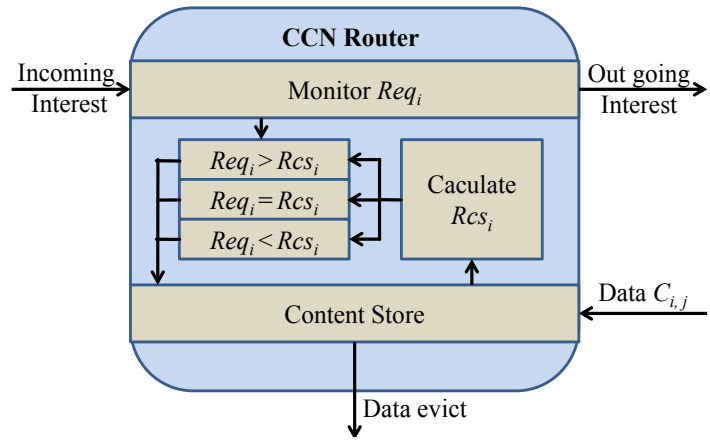

Fig. 1. PPCSA cache replacement policy

When a $\mathrm{CCN}$ router receives a data content $C_{i, j}$, firstly we compare the monitored title-level popularity $\operatorname{Req}_{i}$ and CS storage space occupancy rate $R c_{i}$. And then based on the evictee candidate selection model, we choose the suitable content to evict, as shown in Fig.1.

If $R e q_{i}<R c s_{i}$, video $f_{i}$ is occupying CS rooms more than it needs. Thus some of cache space should be freed for other videos to enhance the CS efficiency. Based on our evictee candidate selection method, we therefore choose two contents $C_{i, p}, C_{i, q}$ of video file $f_{i}$ in the CS to evict, and the evict candidates $C_{i, p}, C_{i, q}$ have the smallest two sequence number ( $p$ $<q$ ), as shown in Fig. 2.

If $R e q_{i}=R c s_{i}$, we select only one content $C_{i, p}$ of video file $f_{i}$ in the $\mathrm{CS}$, which has the minimum sequence number $p$, and evict it.

Otherwise, if $R e q_{i}>R c s_{i}$, it refers that more contents of video $f_{i}$ need to be cached in this CS. Thus no content of video $f_{i}$ will be removed. Instead of that, we select the content $C_{k, p}$ to evict, which has the minimum sequence number $p$ of video $f_{k}$, and this video has the smallest request rate $R e q_{i}$ in CS. Fig.2 illustrate the algorithm.

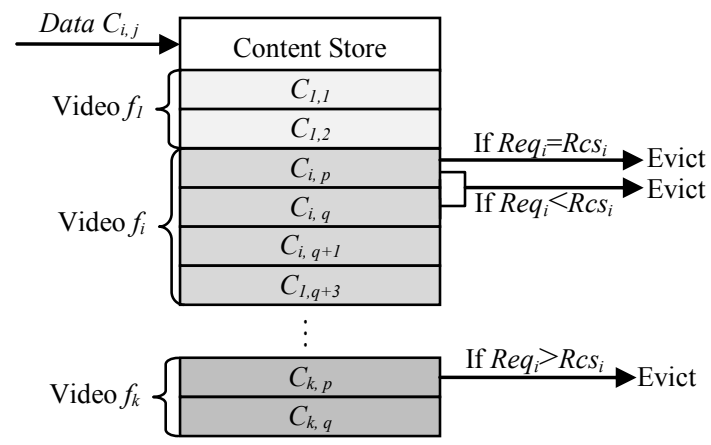

Fig. 2. An example of PPCSA cache replacement algorithm

\section{SimUlation}

We implemented our PPCSA strategies with ndnSIM [12] simulator. We compared our proposed policy with 3 widely adopted chunk-level cache replacement policies LRU, LFU and FIFO on two topologies: a 4-leavel tree topology and a complicated hybrid topology. Leave Copy Everywhere (LCE), the default cache decision policy, has been used in our simulation to work corporately with the cache replacement policies.

\section{A. Simulation configuration}

To evaluate the performance of each cache replacement policy in a realistic network environment, video producers and requesters were all connected to the edge of the topology. And we implement 25 different producers in our simulation, and each of the producers provided a unique video title. In addition, every video file consisted of 800 video chunks. Accordingly the total chunk number is 20,000 items.

Furthermore, referring to title-level popularity, studies have shown that the popularity of video files follows Zipfdistribution [13] [14]. Thus we implemented 100 video viewers and the popularity of request for different video files follows the Zipf-distribution and assumed that $\alpha=1.2$. These viewers started to request videos between a given intervals which were assumed to follows the exponential-distribution. Besides, the consumers requested video contents in the order of the video sequence number $j$ from the beginning of this video to the end. These 100 viewers start to request their target video from different time, and the start time interval of the viewers follows the exponential-distribution. Each simulation was stopped only if all 20,000 contents had been successfully received by their corresponding requesters. And the simulation was performed independently across a range of CS sizes from 100 items to 1000 items for each CCN node.

\section{B. Simulation topologies and evaluation parameters}

We took two topologies to arrange our simulation. The first one is a 4-leavel tree in order to test the performance of our PPCSA policy on a simple and small-scale network. To address the scalability and the performance on complex conditions of PPCSA policy, we carried out another group of simulations arranged on a hybrid topology which composed of a core network and an access network, as shown in Fig.3. We chose GEANT [15] as the core network of our hybrid topology, and for the access networks we used 3-levels complete binary tree.

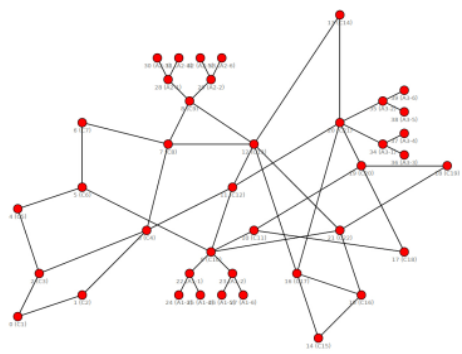

Fig. 3. Hybrid topology 


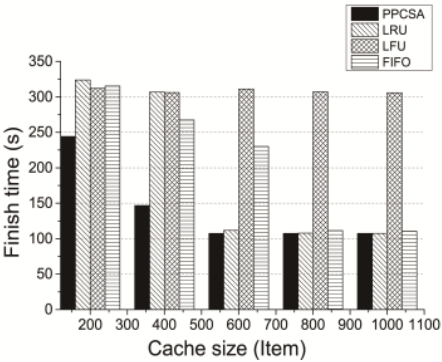

(a) Finish time

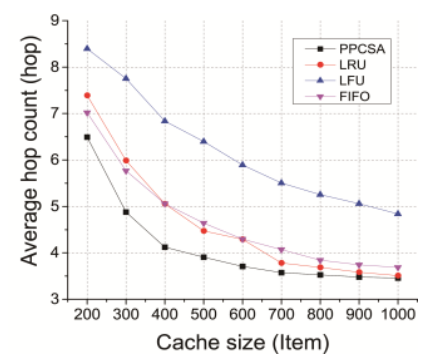

(b) Average hop count

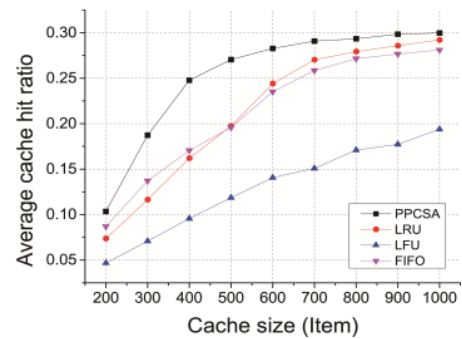

(c) Average cache hit ratio

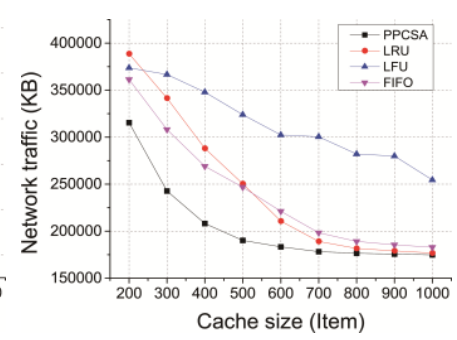

(d) Network traffic

Fig. 4. Results of cache replacement policies on tree topology

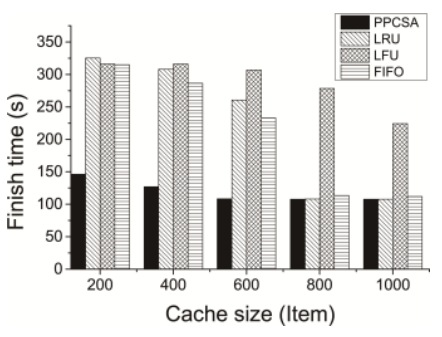

(a) Finish time

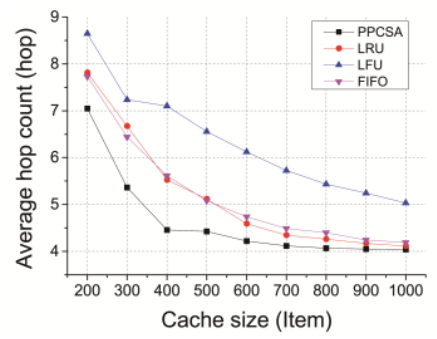

(b) Average hop count

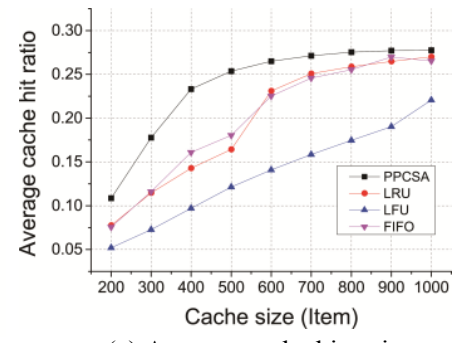

(c) Average cache hit ratio

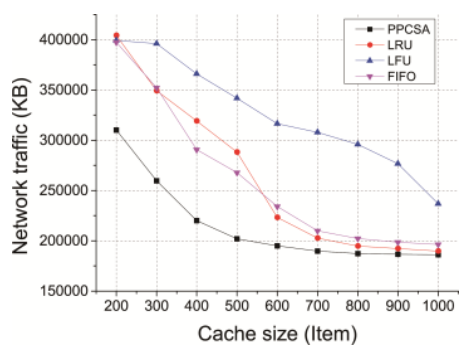

(d) Network traffic

Fig. 5. Results of cache replacement policies on hybrid topology

4 values were used to evaluate the performance of each cache replacement policies.

- Finish time illustrates the total processing time for all the 100 consumers to receive their requested videos contents.

- Average hop count indicates the average transmission round trip distance (number of hops) for each video chunk request.

- Average cache hit ratio is pervasively used, as the primary performance metric to measure the caching performance.

- Network traffic is the sum of total data size which has been forwarded by each node during the entire simulation.

\section{Simulation results}

Results of cache replacement policies arranged on tree topology are presented in Fig. 4. PPCSA reduced the average hop count and raised the average cache hit ratio as shown in Fig. 4 (b) and (c). It denotes that the PPCSA enhances the utilization of cache space, so that viewers can get the video content from the closer routers, in other words, PPCSA lightens the pressure imposed on the original video sources. Furthermore, Fig. 4 (d) provides the evidence that PPCSA policy is more efficient than the other three cache replacement polies, due to unnecessary network traffic reduction.

Therefore, we can conclude that PPCSA policy able to significantly reduce the finish time compared with LRU, LFU and FIFO as shown in Fig. 4 (a). When CS size is more limited, the improvement is more obvious. We believe that there are two possible reasons of the finish time reduction. On one hand, PPCSA reduced the average delivery distance between viewers and target content chunks so that the average transmission time of each video chunk is reduced. On the other hand, by reducing the unnecessary network traffic, the network congestion and packet loss occurred less than other algorithms. Then viewers do not have to frequently resend the request, and thus the total finish time can be reduce obviously.

When PPCSA policy was implemented onto a complex scenario, the improvement of network performances was even more, as shown in Fig. 5. The improvement of finish time and network traffic as shown in Fig. 5 (a) and (d) is more salient than the performance on simple topology. PPCSA preforms independently on each router without further information of other nodes. The video popularity and cache occupancy ratio for each video is based on the real time observation by each independent router, and thus the complexity of network topology cannot affect the performance of PPCSA. Therefore, based on the results, we can denote that the PPCSA policy shows great scalability and also can be deployed to complex network scenarios.

\section{Energy efficiency analysis}

It can be observed that many studies argued the power consumption of CCN [16] [17]. Researchers are currently making their efforts to reduce the energy consumption of CCN in different aspects. In our study, according to the simulation 
results, a power consumption model has been proposed, as shown below.

$$
\begin{gathered}
E_{C C N}=E_{t}+E_{s}+E_{\text {idle }} \\
E_{t}=P_{t} \times \text { Network Traffic } \\
E_{s}=P_{s} \times \text { Total Cache Size } \\
E_{\text {idle }}=P_{\text {idle }} \times \text { Finish Time }
\end{gathered}
$$

The total power consumption of $\mathrm{CCN}$ expressed as $E_{C C N}$ has three components, as shown in (3): $E_{t}$ indicates the transmission power consumption by $\mathrm{CCN}$, and $P_{t}(\mathrm{~J} / \mathrm{GB})$ represents the energy per size unit to transport data across the network. $E_{s}$ refers to the storage energy cost while $P_{s}(\mathrm{~J} / \mathrm{GB})$ is the cache energy consumed per GB. And the $E_{\text {idle }}$ refers to the static power consumed by the network during the whole simulation, and $P_{\text {idle }}(\mathrm{J} / \mathrm{s})$ represents the idle network power consumption per second.

We can observe from our results that by reducing the finish time, PPCSA is capable of reducing the $E_{i d l e}$. On the other hand, transmission power $E_{t}$ can be cut down according to the reduction of network traffic. And the reduction percentage is shown in Fig. 6. PPCSA reduces the transmission energy consumption over $15 \%$ on average compared to LRU and FIFO, and nearly $30 \%$ to LFU. And for $E_{\text {idle, }}$ the reductions reach $34 \%$ compare to LRU and FIFO, and $60 \%$ from LFU.

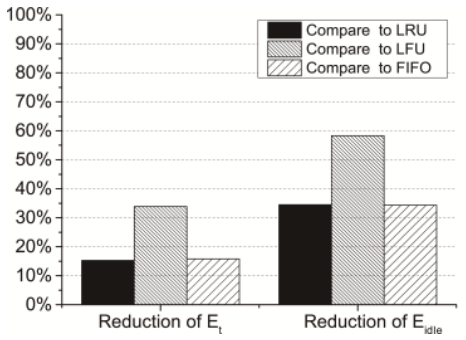

Fig. 6. Power reduction percentage of PPCSA compare to LRU, LFU and FIFO

\section{CONCLUSION}

By considering the characteristics of video delivery, we proposed a cache replacement policy PPCSA which is an effort to enhance the cache performance of $\mathrm{CCN}$. In this paper, we introduced our PPCSA model and policy with both title- and content-level popularity considered. In our simulation, we implemented PPCSA policy in ndnSIM simulator as well as LRU, LFU and FIFO for comparison. And the results show that on both tree and hybrid topologies, PPCSA increased the cache hit ratio, shortened the average communication time, and average contents delivery distance between requesters and video contents, and showed great scalability and adaptability for complex network scenarios. In addition, we analyzed the energy efficiency of above mentioned cache replacement policies and the result shows that PPCSA is more energy efficient than other three cache replacement policies.

In the future study, we intend to focus on improving the practicability and the energy saving potential of PPCSA, as well as the accuracy of evictee candidate selection which according to the predict-based future popularity.

\section{ACKNOWLEDGMENT}

The work for this paper was performed in the context of the FP7/NICT EU-JAPAN GreenICN project.

\section{REFERENCES}

[1] "Cisco Visual Networking Index: Forecast and Methodology, 20132018 White paper", June 10, 2014.

[2] Koponen T, Chawla M, Gon C B, et al.. "A data-oriented (and beyond) network architecture," ACM SIGCOMM 2007 Conference, Kyoto, Japan, pp. 181-192, 2007.

[3] N. Fotiou, P. Nikander, D. Trossen, G.C. Polyzos, Developing Information Networking Further: From PSIRP to PURSUIT, International ICST Conference on Broadband Communications, Networks, and Systems (BROADNETS), October 2010

[4] European Union. Project FP7 4WARD. http://www. 4ward-project.eu, 2010.

[5] V. Jacobson, D. K. Smetters, J. D. Thornton, M. F. Plass, N. H. Briggs, and R. L. Braynard, "Networking named content," in Proceedings of the 5 th international conference on Emerging networking experiments and technologies. ACM, 2009, pp. 1-12.

[6] Y. Li, H. Xie, Y. Wen, Z. Zhang, "Coordinating In-Network Caching in Content-Centric Networks: Model and Analysis," ICDCS 2013 IEEE 33rd International Conf., pp. 62-72, Philadelphia, USA, Jul. 2013.

[7] Psaras, Ioannis, Wei Koong Chai, and George Pavlou. "Probabilistic innetwork caching for information-centric networks." Proceedings of the second edition of the ICN workshop on Information-centric networking. ACM, 2012.

[8] M. Draxler and H. Karl, "Efficiency of on-path and off-path caching strategies in information centric networks," Proc. of the IEEE International Conf. on Green Computing and Commun. (GreenCom '12), pp. 581-587, Besancon, France, Nov. 2012.

[9] Lee, Junghwan, Kyubo Lim, and Chuck Yoo. "Cache replacement strategies for scalable video streaming in CCN." Communications (APCC), 2013 19th Asia-Pacific Conference on. IEEE, 2013.

[10] Wu, Tingyao, et al. "Reuse time based caching policy for video streaming." Consumer Communications and Networking Conference (CCNC), 2012 IEEE. IEEE, 2012.

[11] Belady L A. A study of replacement algorithms for a virtual-storage computer[J]. IBM Systems journal, 1966, 5(2): 78-101.

[12] A. Afanasyev, I. Moiseenko, and L. Zhang, "ndnSIM: NDN simulator for NS-3," NDN, Technical Report NDN-0005, 2012.

[13] P. Gill, M. Arlitt, Z. Li, and A. Mahanti, "Youtube traffic characterization:a view from the edge", Proc. of the 7 th ACM SIGCOMM Conf., pp.15-28, 2007.

[14] Cha, Meeyoung, et al. "I tube, you tube, everybody tubes: analyzing the world's largest user generated content video system." Proceedings of the 7th ACM SIGCOMM conference on Internet measurement. ACM, 2007.

[15] “GÉANT Project [on-line]" http://www.geant.net/.

[16] Imai, Satoshi, Kenji Leibnitz, and Masayuki Murata. "Energy-aware cache management for content-centric networking." Advanced Information Networking and Applications Workshops (WAINA), 2013 27th International Conference on. IEEE, 2013.

[17] Hasegawa, Toru, et al. "Empirically modeling how a multicore software ICN router and an ICN network consume power." Proceedings of the 1st international conference on Information-centric networking. ACM, 2014. 